\title{
OPENNESS OF THE DERIVATIVE OF A COMPLEX FUNCTION
}

\author{
ROBERT L. PLUNKETT ${ }^{1}$
}

1. Introduction. The principal object of this paper is to present a purely topological proof of the strong openness of the derivative of a complex function. This openness is, of course, a direct consequence of the existence of the second derivative, but the proof of that existence still requires the use of powerful techniques of function theory which depend on complex integration. The proof presented here is therefore necessarily independent of the existence of the second derivative.

Heavy use is made of the results and techniques described in Topological analysis, by G. T. Whyburn [1]. The continuity of the derivative, an elementary proof of which has recently been given [2], is also used extensively in the proofs, both directly and in conjunction with the topological index [1, Chapter V].

In the following, $f$ will denote a nonconstant complex valued function defined and differentiable in a region (connected, open set) $E$ of the complex plane. The main theorem is proved in two ways, both of which depend on the uniform differentiability of $f$ on compact subsets of $E$ (Theorem 1). The first approach is the more economical, obtaining the conclusion by the direct application of two theorems of Whyburn's. The second approach is also presented since it is possible that some of the intermediate steps are of interest in themselves.

2. Uniform differen'iabiity. The function $f$ is said to be uniformly differentiable on the subset $A$ of $E$ if and only if, for each $\epsilon>0$, there exists a $\delta>0$ such that $z \in A, z^{\prime} \in E$ and $0<\left|z^{\prime}-z\right|<\delta$ imply that

$$
\left|\frac{f\left(z^{\prime}\right)-f(z)}{z^{\prime}-z}-f^{\prime}(z)\right|<\epsilon .
$$

A discussion of uniform differentiability from the function theoretic point of view may be found in [3, p. 179].

Presented to the Society, November 21, 1959; received by the editors November 9, 1959.

${ }^{1}$ Research supported by the United States Air Force Office of Scientific Research of the Air Research and Development Command, under Contract No. AF49(638)-598. Reproduction in whole or in part is permitted for any purpose of the United States Government. 
THEOREM 1. The function $f$ is uniformly differentiable on each compact subset $D$ of $E$.

ProOF. If the conclusion fails, then there exists an $\epsilon>0$ such that, for each positive integer $i$, there exists a $z_{i} \in D$ and a $z_{i}^{\prime} \in E$ such that $z_{i} \neq z_{i}^{\prime},\left|z_{i}-z_{i}^{\prime}\right|<1 / i$, and

$$
\left|\frac{f\left(z_{i}^{\prime}\right)-f\left(z_{i}\right)}{z_{i}^{\prime}-z_{i}}-f^{\prime}\left(z_{i}\right)\right| \geqq \epsilon .
$$

The sequence $\left\{z_{i}\right\}$ being obviously infinite, let $z_{0}$ denote one of its limit points in $D$ and suppose that by choosing a subsequence converging to $z_{0}$ and renumbering it we have arranged that $z_{i} \rightarrow z_{0}$. The corresponding primed sequence $\left\{z_{i}^{\prime}\right\}$ also clearly converges to $z_{0}$.

For each $i$, let $N_{i}(z)$ be the continuous function defined in $E$ by

$$
N_{i}(z)=\left\{\begin{array}{cl}
\frac{f(z)-f\left(z_{i}\right)}{z-z_{i}}-f^{\prime}\left(z_{i}\right), & \text { if } z \neq z_{i}, \text { and } \\
0, & \text { if } z=z_{i} .
\end{array}\right.
$$

Also let $\left\{E_{i}\right\}$ be a sequence of open two cell neighborhoods of $z_{0}$, each contained in $E$ and such that diam $E_{i} \rightarrow 0$, as $i \rightarrow \infty$. We may assume that each $E_{i}$ contains both $z_{i}$ and $z_{i}^{\prime}$, since this can be arranged by taking subsequences. Now, on $E_{i}, N_{i}(z)$ is continuous and we have $N_{i}\left(z_{i}\right)=0$ and $\left|N_{i}\left(z_{i}^{\prime}\right)\right| \geqq \epsilon$. That is, each connected set $N_{i}\left(E_{i}\right)$ contains both zero and a point whose modulus is $\geqq \epsilon$. Hence each $E_{i}$ contains a point $z_{i}^{*}$ such that $N_{i}\left(z_{i}^{*}\right)=\alpha_{i}$, where $\left|\alpha_{i}\right|=\epsilon / 2$. Also, of course, $z_{i}^{*} \rightarrow z_{0}$.

Suppose $\left\{\alpha_{i}\right\}$ is an infinite sequence. Then, since all $\alpha_{i}$ belong to the compact set $\{z:|z|=\epsilon / 2\}$, there exists a limit point $\alpha$ such that $|\alpha|=\epsilon / 2$. By taking subsequences here and back down the line, we may suppose that $\alpha_{i} \rightarrow \alpha$. Now let $g(z)$ denote the function $f(z)$ $-\left[f^{\prime}\left(z_{0}\right)+\alpha\right] z$ and observe that, since $g^{\prime}\left(z_{0}\right)=-\alpha \neq 0, g$ is a local homeomorphism at $z_{0}[1$, p. 85]. Using this fact, suppose that $H$, with boundary $C$, is an open circular neighborhood of $z_{0}$ with $H \cup C$ contained in $E$ and such that $g$ is a homeomorphism on $H \cup C$.

For each $i$, let $F_{i}(z)$ denote the function $g(z)-g\left(z_{i}\right)$ and let $G_{i}(z)$ denote the function $\left[\left(f^{\prime}\left(z_{0}\right)+\alpha\right)-\left(f^{\prime}\left(z_{i}\right)+\alpha_{i}\right)\right]\left(z-z_{i}\right)$. If $F(z)$ temporarily denotes $g(z)-g\left(z_{0}\right)$, then $\min _{z \in C}|F(z)|=d>0$, since $0 \notin F(C)$. Now, since $\left\{F_{i}\right\}$ converges uniformly on $C$ to $F$, there exists an $I_{1}$ such that $i>I_{1}$ implies that $\min _{z \in C}\left|F_{i}(z)\right|>2 d / 3$.

By the continuity of $f^{\prime}$ on $E[2]$, we know that $\left\{f^{\prime}\left(z_{i}\right)+\alpha_{i}\right\}$ converges to $\left(f^{\prime}\left(z_{0}\right)+\alpha\right)$ and, since $z_{i} \rightarrow z_{0}$, it can be seen that there exists 
an $I_{2}$ such that $i>I_{2}$ implies that $\left|G_{i}(z)\right|<d / 3$, for all $z \in C$. Finally, there exists an $I_{3}$ such that $i>I_{3}$ implies that both $z_{i}$ and $z_{i}^{*}$ belong to $H$.

Fix $i$ at a value greater than all of $I_{1}, I_{2}$, and $I_{3}$. Then $\left|G_{i}(z)\right|$ $<\left|F_{i}(z)\right|$, for all $z \in C$. By Rouché's theorem $\left[1\right.$, p. 93], $F_{i}(z)+G_{i}(z)$ has exactly the same number of zeros in $H$ as $F_{i}(z)$, which, of course, has only one, since $g$ is a homeomorphism on $H$. But

$$
\begin{aligned}
F_{i}(z)+G_{i}(z)= & g(z)-g\left(z_{i}\right)+\left[\left(f^{\prime}\left(z_{0}\right)+\alpha\right)-\left(f^{\prime}\left(z_{i}\right)+\alpha_{i}\right)\right]\left(z-z_{i}\right) \\
= & f(z)-f\left(z_{i}\right)-\left[\left(f^{\prime}\left(z_{0}\right)+\alpha\right)\right]\left(z-z_{i}\right) \\
& +\left[\left(f^{\prime}\left(z_{0}\right)+\alpha\right)-\left(f^{\prime}\left(z_{i}\right)+\alpha_{i}\right)\right]\left(z-z_{i}\right) \\
= & f(z)-f\left(z_{i}\right)-\left[\left(f^{\prime}\left(z_{i}\right)+\alpha_{i}\right)\right]\left(z-z_{i}\right),
\end{aligned}
$$

which equals zero when $z=z_{i}$ and, if $z \neq z_{i}$, then

$$
F_{i}(z)+G_{i}(z)=\left[N_{i}(z)-\alpha_{i}\right]\left(z-z_{i}\right),
$$

which equals zero when $z=z_{i}{ }^{*}$. Thus we have arrived at a contradiction in case $\left\{\alpha_{i}\right\}$ is an infinite sequence. A similar (easier) proof provides a contradiction in case $\left\{\alpha_{i}\right\}$ is finite.

3. Openness: First method. An immediate consequence of Theorem 1 is:

CoROllary 1.1. Let $\left\{a_{i}\right\}$ be any sequence of nonzero complex numbers converging to 0 . Then, for each compact subset $D$ of $E$, there exists a positive integer $I$ such that, for each $i \geqq I$, the mapping $h_{i}$ defined by

$$
h_{i}(z)=\frac{f\left(z+a_{i}\right)-f(z)}{a_{i}}
$$

is defined on $D$ and the sequence $\left\{h_{i}: i \geqq I\right\}$ converges uniformly on $D$ to the mapping $f^{\prime}$.

The main result of this paper is now available.

CoROllary 1.2. If $f^{\prime}$ is not constant on $E$, then it is a strongly open mapping of $E$ into the complex plane.

Proor. By $\left[1\right.$, p. 89], $f^{\prime}$ is light. By the preceding corollary and another theorem of G. T. Whyburn's [1, p. 113], trivially modified, $f^{\prime}$ is quasi-open. But these conditions on $f^{\prime}$ ensure that it is strongly open.

4. Openness: Second method. Theorems 2, 3, and 4, which will be proved in this section, are presented here because of their similarity to known results concerning differentiable functions. 
THEOREM 2. If $f^{\prime}$ is not constant on $E$ and if $f^{\prime}\left(z_{0}\right)=0$, for $z_{0} \in E$, then, for each sufficiently small simple closed curve $C$ containing $z_{0}$ in its interior,

$$
(1 / 2 \pi i) \mu_{C}\left(f^{\prime}, 0\right) \geqq 1 .
$$

Proof. It is known under these conditions that $f^{\prime}$ has scattered point inverses [1, p. 89]; i.e., for each $w \in f^{\prime}(E)$ each point of $\left(f^{\prime}\right)^{-1}(w)$ is an isolated point of $\left(f^{\prime}\right)^{-1}(w)$. Hence there exists in $E$ an open circular disk $D$ centered at $z_{0}$ such that $z \in D-\left\{z_{0}\right\}$ implies that $f^{\prime}(z) \neq 0$. Now let $C$ be any simple closed curve in $D$ containing $z_{0}$ in its interior, $R$.

Since $f$ fails to be a local homeomorphism at $z_{0}[1$, p. 85$]$, there exists a pair $\left\{z_{i}\right\},\left\{z_{i}^{*}\right\}$ of $f$-companion sequences (see [2]) in $R$ converging to $z_{0}$. If $t_{i}$ denotes $z_{i}^{*}-z_{i}$, for each $i$, then there is a positive integer $I_{1}$ such that $i>I_{1}$ implies that $z+t_{i} \in E$ for all $z \in R \cup C$. Also there exists an integer $I_{2}>I_{1}$ such that $i>I_{2}$ implies that $f\left(z+t_{i}\right)-f(z)=0$ for no $z \in C$. For, otherwise, there would exist a pair of $f$-companion sequences in $E$ converging to a point $z^{*} \in C$ and $f^{\prime}\left(z^{*}\right)$ would consequently equal zero [2], contradicting the selection of $C$.

For each $i>I_{2}$, the function $g_{i}(z)=f\left(z+t_{i}\right)-f(z)$ is then differentiable in $R \cup C, g_{i}\left(z_{i}\right)=0$, but $g_{i}(z)=0$ for no $z \in C$. Hence $0 \in g_{i}(R \cup C)$ $-g_{i}(C)$. Now for any positive traversal of $C$, by $[1, \mathrm{p} .73]$, we have $(1 / 2 \pi i) \mu_{C}\left(g_{i}, 0\right) \geqq 1$, for each $i>I_{2}$.

To get the same result for $f^{\prime}$, let $h_{i}(z)=g_{i}(z) / t_{i}$, for each $i>I_{2}$ and all $z \in C$, and let $d=\min _{z \in C}\left|f^{\prime}(z)\right|$. Since $f^{\prime}$ is continuous and $C$ is compact, $d>0$. By Corollary 1.1, there then exists an $I_{3}$ such that for all $i>I_{3}$,

$$
\left|h_{i}(z)-f^{\prime}(z)\right|<d \leqq\left|f^{\prime}(z)-0\right|, \quad \text { for all } z \in C .
$$

Hence, by $[1$, p. 65$]$, for $i>I_{3}$,

$$
\mu_{C}\left(h_{i}, 0\right)=\mu_{C}\left(f^{\prime}, 0\right) .
$$

Fix $i$ at a value larger than both $I_{2}$ and $I_{3}$. Then, since $g_{i}(z)$ $=t_{i} h_{i}(z)$, for all $z \in C$, we have $[1$, p. 58] that

$$
\mu_{C}\left(g_{i}, 0\right)=\mu_{C}\left(t_{i}, 0\right)+\mu_{C}\left(h_{i}, 0\right) .
$$

But $\mu_{C}\left(t_{i}, 0\right)$ is zero, since $t_{i}$ is constant, and, since $(1 / 2 \pi i) \mu_{C}\left(g_{i}, 0\right) \geqq 1$, we have $(1 / 2 \pi i) \mu_{C}\left(h_{i}, 0\right) \geqq 1$. By $(\dagger)$, then, $(1 / 2 \pi i) \mu_{C}\left(f^{\prime}, 0\right) \geqq 1$.

THEOREM 3. Let $f(z)$ be a complex valued function defined and having a nonconstant derivative in $E$. If $C$ is a simple closed curve contained 
with its interior $R$ in $E$ and if $p \in f^{\prime}(R \cup C)-f^{\prime}(C)$, then

$$
(1 / 2 \pi i) \mu_{C}\left(f^{\prime}, p\right) \geqq 1 \text {. }
$$

Proof. $(R \cup C) \cap f^{\prime-1}(p)$ is a finite set $\left\{z_{1}, z_{2}, \cdots, z_{n}\right\}$, since $f^{\prime-1}(p)$ is scattered, and $z_{j} \notin C$ for any $j \in\{1,2, \cdots, n\}$, since $p=f^{\prime}\left(z_{j}\right)$ $\notin f^{\prime}(C)$. Replacing $f(z)$ temporarily by the function $g(z)=f(z)-p z$, note that $\left\{z_{1}, z_{2}, \cdots, z_{n}\right\}=g^{\prime-1}(0) \cap(R \cup C)$ and that $0 \notin g^{\prime}(C)$. Employing Theorem 2 , we may construct circles $C_{1}, C_{2}, \cdots, C_{n}$, centered at $z_{1}, z_{2}, \cdots, z_{n}$, respectively, and sufficiently small that each plus its interior is contained in $R$, no two of them or their interiors intersect, and, for each $j \in\{1,2, \cdots, n\},(1 / 2 \pi i) \mu_{C j}\left(g^{\prime}, 0\right)$ $\geqq 1$.

By the additivity of the topological index, we have

$$
(1 / 2 \pi i) \mu_{C}\left(g^{\prime}, 0\right)=\sum_{j=1}^{n}(1 / 2 \pi i) \mu_{C j}\left(g^{\prime}, 0\right) \geqq n \geqq 1,
$$

since $g^{\prime}(z)=0$ for no $z$ in the region between $C$ and the collection $C_{1}, \cdots, C_{n}$. But $\mu_{C}\left(g^{\prime}, 0\right)=\mu_{C}\left(f^{\prime}-p, 0\right)=\mu_{C}\left(f^{\prime}, p\right)$, by $[1$, p. 58], completing the proof.

THEOREM 4. For each simple closed curve $C$ contained with its interior, $R$, in $E, f^{\prime}(R \cup C)$ consists of $f^{\prime}(C)$ together with a collection of bounded complementary domains of $f^{\prime}(C)$.

Proof. For each $p \in f^{\prime}(R \cup C)-f^{\prime}(C)$ we have $(1 / 2 \pi i) \mu_{C}\left(f^{\prime}, p\right)>0$; hence, $p \notin$ the unbounded complementary component of $f^{\prime}(C)$. On the other hand, let $\Omega$ be a bounded complementary component of $f^{\prime}(C)$ which intersects $f^{\prime}(R \cup C)$. For each $p$ in the intersection, $(1 / 2 \pi i) \mu_{C}\left(f^{\prime}, p\right)>0$. If $\Omega$ contained a point $q$ not in $f^{\prime}(R \cup C)$, then $(1 / 2 \pi i) \mu_{C}\left(f^{\prime}, q\right)$ would equal zero $[1$, p. 68] contradicting the constancy of the index on $\Omega\left[1\right.$, p. 59]. Hence $\Omega \subset f^{\prime}(R \cup C)$.

Because of Theorem 4 the second proof of Corollary 1.2 may now be obtained by copying the proof of Theorem (5.1) of $[1$, p. 76], replacing $f$ by $f^{\prime}$.

\section{REFERENCES} 1958.

1. G. T. Whyburn, Topological analysis, Princeton, Princeton University Press,

2. R. L. Plunkett, $A$ topological proof of the continuity of the derivative of a function of a complex variable, Bull. Amer. Math. Soc. vol. 65 (1959) pp. 1-4.

3. E. Hille, Analytic function theory, Volume 1, Ginn and Company, 1959.

The Florida State University 